\title{
An OFDMA-based Hybrid MAC Protocol for IEEE 802.11ax
}

\author{
Gazi Zahirul Islam and Mohammod Abul Kashem
}

\begin{abstract}
Two types of MAC mechanisms i.e., random access and reservation could be adopted for OFDMA-based wireless LANs. Reservation-based MAC is more appropriate than random access MAC for connection-oriented applications as connectionoriented applications provide strict requirements of traffic demands. On the other hand, random access mechanism is a preferred choice for bursty traffic i.e., data packets which have no fixed pattern and rate. As OFDMA-based wireless networks promise to support heterogeneous applications, researchers assume that applications with and without traffic specifications will coexist. Eventually, OFDMA-based wireless LAN will deploy hybrid MAC mechanisms inheriting traits from random access and reservation. In this article, we design a new MAC protocol which employs one kind of hybrid mechanism that will provide high throughput of data as well as maintains improved fair access policy to the medium among the terminals. The protocol works in two steps, where at step 1 sub-channels are approximately evenly distributed to the terminals and at step 2 terminals within in a subchannel will contend for medium randomly if the total number of terminals of the system is larger than the number of sub-channels. The details of the protocol is illustrated in the paper and we analyze the performance of our OFDMA-based multi-channel hybrid protocol using comprehensive computer simulations. Simulation results validate that our proposed protocol is more robust than the conventional CSMA/CA protocol in terms of throughput, collision reduction and fair access. In addition, the theoretical analysis of the saturation throughput of the protocol is also evaluated using an existing comprehensive model.
\end{abstract}

Index Terms-Throughput, MAC, OFDMA, IEEE 802.11ax, CSMA/CA, Wi-Fi 6.

\section{INTRODUCTION}

The rapid growth of demand for high-speed WLAN has driven the exhaustive research to enhance the throughput by employing a variety of medium access control (MAC) mechanisms. The efficiency of MAC plays a major role to enhance the throughput of any wireless LAN system. One of the innovative and promising access procedures for MAC is orthogonal frequency division multiple access (OFDMA) which originally derived from orthogonal frequency division multiplexing (OFDM). An OFDMA system uses a group of non-overlapping sub-carriers to form a sub-channel that can be allocated to each transmitting station. Thus, multiple stations

Gazi Zahirul Islam is currently pursuing his $\mathrm{PhD}$ at Bangladesh University of Professionals, Dhaka-1216, Bangladesh. He is also teaching at Department of Computer Science and Engineering, Daffodil International University, Bangladesh (e-mail: zahircuet@gmail.com).

Mohammod Abul Kashem is a Professor of Department of Computer Science and Engineering, Dhaka University of Engineering and Technology, Gazipur-1700, Bangladesh (email: drkashemll@duet.ac.bd) can send data concurrently without having collision [1]. Absorbing the advantages of OFDM, OFDMA-based MAC protocol can further enhance efficiency by increasing multiuser diversity. As such superiority of OFDMA technology, some wireless systems such as WiMAX leverages it from the very beginning.

According to the functional requirements of IEEE 802.11ax, Wi-Fi should achieve at least 4 times improvement in the average throughput per station (terminal) as well as should support highly dense systems [14]. The physical data rate in Wireless LAN has been remarkably boosted due to more available bandwidth resources and the arrival of modern technologies such as MIMO [5]. However, the MAC layer of Wireless LANs has not changed significantly for the last 16 years. Since their birth, Wireless LANs employed distributed coordination function (DCF) as the MAC layer protocol [19]. According to the DCF protocol, only one station can utilize the channel resource and send data at the same time [9]. DCF rules employed in IEEE 802.11 are suited to sparsely dense Wireless LAN environment, while in the highly dense system the MAC efficiency of DCF would be very poor due to the provision of single user accessibility [15]. To overcome the difficulties mentioned above, multiuser MAC is required instead of a single user [16]. Since Wireless LANs have already included OFDM as modulation technology, OFDMA technology is highly recommended for next generation Wireless LANs [17]. An OFDM adopted system enables a single terminal to utilize all the sub-channels at any given time while an OFDMA adopted system enables multiple terminals to use a different set of subchannels, thereby providing concurrent transmission of more than one terminal [2].

Two types of MAC mechanisms namely, random access and reservation can be employed for OFDMA-based wireless LANs. Reservation-based MAC is more appropriate than random access MAC for connection-oriented applications as connection-oriented applications provide clear specifications of traffic demands. Reservation-based MAC ensures graceful support for Quality of Service (QoS). However, it is not appropriate for applications that contain no traffic specifications. For example, in data networks like the Internet, an application is usually characterized by bursty traffic, i.e., data packets arrive in an arbitrary pattern and rate. So, it would be unwise to reserve a certain amount of resources (e.g., subchannels in OFDMA) for applications in a data network. Thus, random access mechanism is a preferred choice for bursty traffic. As OFDMA-based wireless networks promise to support heterogeneous applications, it is anticipated that applications with clear traffic specifications and those without traffic specifications will coexist [3]. To this end, both 
reservation-based and random-access MAC procedures are optimized for an OFDMA-based wireless LAN. In other words, the MAC protocol of an OFDMA-based wireless network will provide a hybrid MAC mechanism for both random access and reservation.

In this paper, we propose an innovative MAC protocol which employs a hybrid mechanism that could provide high throughput of data as well as able to maintain improved fair access policy to the medium among the terminals. The protocol works in two steps, where at step 1 sub-channels are approximately evenly distributed to the terminals and at step 2 terminals within in a sub-channel will contend for medium randomly if the total number of terminals of the system is larger than the number of sub-channels. The details of the protocol will be described in the 'Protocol Illustration' section i.e. Section IV.

The rest of the article is organized as follows. At first, we discuss related works and motivation in Section II. Section III contains the system model and Section IV contains protocol illustration. Mathematical analysis of the saturation throughput of the protocol is evaluated in Section V using a comprehensive model. Simulation is conducted by renowned 'NS-3 Simulator' [10] and presented the result in Section VI. Finally, Section VII concludes the paper.

\section{RELATED Works AND MotiVATION}

Recently, there has been rigorous research devoted to the combination of OFDMA with MAC. Xuelin et al. in [6] designed a multi-step slot reservation hybrid MAC protocol named 'TR-MAC' for ad hoc networks which incorporates the strengths of TDMA (Time Division Multiple Access) and DCF of IEEE 802.11. TR-MAC eliminates the slot assignment algorithm, reduces the control packets negotiation and avoids extra contentions. Thus, enhanced the throughput of MAC without incurring additional overhead. The researchers in [7] devised a model named 'CCRM' which innovates a new asynchronous MAC protocol with cooperative channel reservation. Compared with legacy channel reservation MAC protocols, where channel reservation information (CRI) cannot be obtained reliably due to either transmission errors or packet collisions, CCRM improves the reliability of channel reservation using cooperative channel reservation mechanism.

Several random access protocols have been designed for OFDMA-based WLANs. The authors of [8] and [3] proposed a protocol using a two-dimensional backoff scheme to enable the terminals accessing the channel both in the time and frequency domains. The articles [20] and [21] divide stations into multiple groups and the stations in the same group share the same subchannel for channel access. Once the access point receives an RTS (request-to-send) frame from the sub-channels, it replies with a CTS (clear-to-send) frame to assign the channel resources.

Choi et al. [8] put forward an innovative fast retrial slotted ALOHA-based scheme to reduce access delay, but the throughput of the protocol is very poor due to high collision probability. In article [2], the researchers designed a random access model based on the CSMA/CA technique that outperforms traditional ALOHA protocol. According to that model, a terminal employs only one backoff timer for all the sub-channels, and the timer could not reflect different traffic loads in different sub-channels. As a result, the channel utilization efficiency of the model in [2] is still not satisfactory. This constraint is then resolved by Wang in [3], where a terminal employs one backoff timer for each of the sub-channel. Therefore, the transmission status of one sub-channel does not affect the rest of the sub-channels. To overcome the half-duplex limitation of the wireless radio the authors of [3] proposed utilizing an additional radio to sense the medium on all other sub-channels while the original radio is busy in transmission on a certain sub-channel. Thus, the scheme improved transmission concurrency on multiple sub-channels. However, this sort of scheme having a dedicated sensing module is not applicable to the station with a single radio. Jia $\mathrm{Xu}$ et al. [4] introduce intermittent carrier sense technique that permits a single-radio OFDMA station to access multiple sub-channels simultaneously. However, the total throughput of the system and the max-min fairness is not yet satisfactory to meet the demand of IEEE 802.11ax network.

Considering above ideas and facts, we design a new MAC protocol named 'HTFA' for high throughput and fair access. The main contribution of HTFA is as follow:

- One of the major goals of IEEE 802.11ax (Wi-Fi 6) is to improve the total system throughput as well as per terminal throughput. HTFA provides higher throughput than several promising protocols which will be described in the 'Performance Evaluation and Simulation' section.

- HTFA leverages hybrid mechanisms to distribute channel access time more evenly among the terminals. Thus it ensures fair access policy and performs better than SRMC-CSMA/CA and CM-CSMA/CA introduced in [4] and [3] respectively.

- The terminals in HTFA will not contend for sub-channel access if the number of terminals is smaller or equal to the number of sub-channels. Thus, the probability of frame collision is zero. Since there is no backoff slot, there is no idle slot as well. Hence, system throughput increases significantly.

- We perform an extensive simulation with network simulator NS-3 [10] which is presented in Section VI. Simulation results confirm validation of our protocol in terms of throughput, collision reduction and fairness.

\section{SYSTEM MODEL}

We consider an OFDMA-employed WLAN where total bandwidth $B$ is equally distributed to $M$ sub-channels. Hence the bandwidth of a sub-channel would be $B / M$. There are $N$ stations and only one access point (AP) in our system. By choosing different sub-channels, more than one station can communicate with the AP at the same time without suffering from co-channel interference. In such a network collision would occur if and only if multiple stations send packets on the same sub-channel concurrently.

The 802.11ax standard hires some technological developments from $4 \mathrm{G}$ cellular technology to support more stations in the same channel bandwidth leveraging OFDMA. 802.11ax not only adopted OFDM digital modulation scheme but also allocates a group of non-overlapping subcarriers to individual stations. The standard partitions the existing 802.11 channels which may be $20 / 40 / 80 / 160 \mathrm{MHz}$ wide into smaller 


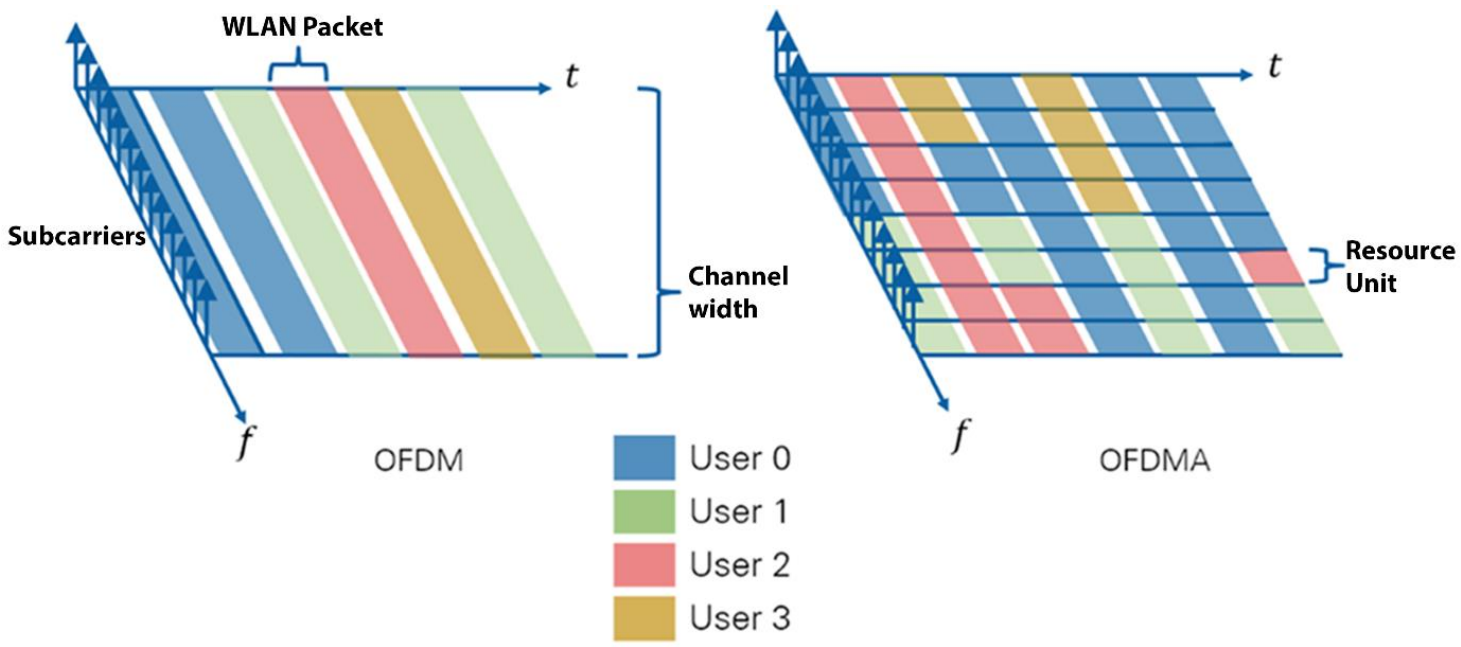

Fig. 1. The contrast between OFDM and OFDMA

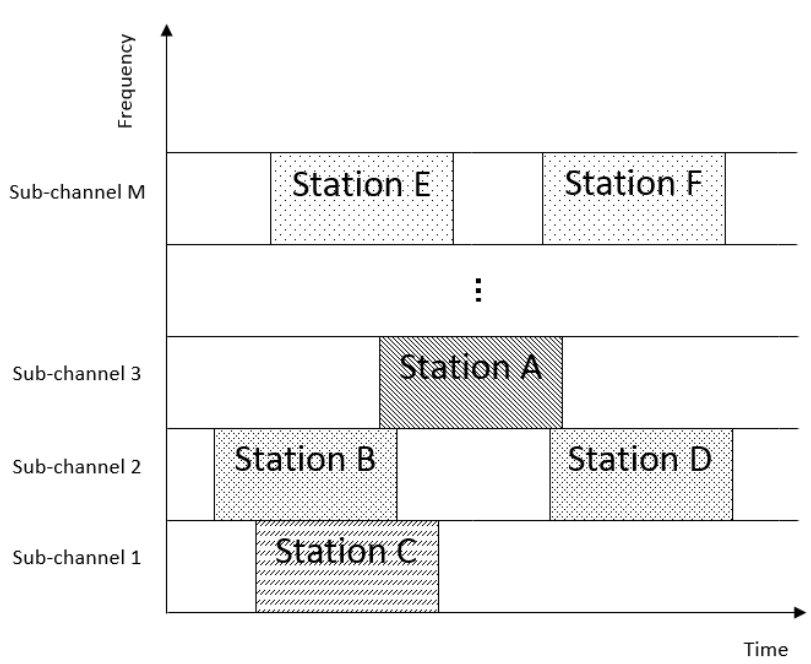

Fig. 2. A 2-dimensional time-frequency access model

sub-channels with a specified number of orthogonal subcarriers [18]. Following LTE (Long Term Evolution) nomenclature, the 802.11ax standard terms the smallest subchannel as a resource unit (RU) which contains at least 26 subcarriers.

Observing different user's traffic needs, the access point decides on allocating the channel, always allocating available resource units on the downlink path. The AP might assign the whole channel to only one terminal or it may divide the channel to form sub-channels for serving multiple terminals simultaneously (Fig. 1). In congested areas where lots of terminals would normally compete inefficiently for channel access, the OFDMA technology can now serve them concurrently with a smaller but dedicated sub-channel. As a result, the average throughput per user is enhancing significantly.
As mentioned earlier, in comparison with the single-channel CSMA/CA, the multi-channel system facilitates stations to access the sub-channels simultaneously without having interference. In a multi-channel system, stations can compete for available resources from both time and frequency domain as shown in Fig. 2 [22]. In the time domain, stations could acquire the time slots of a sub-channel when the sub-channel is not busy and avoid possible collision (if more than one station present) using binary exponential backoff (BEB) algorithm. In the frequency domain, stations could use different sub-channels concurrently and prevent interference using OFDMA mechanism.

We suppose every station maintains its own timer and synchronizes its timer with other station's timer. To ensure clock synchronization, the AP informs the reference time information to participating stations at a regular interval according to the time synchronization function suggested by the IEEE 802.11 standard [9]. It is noted that imperfect synchronization generates clock offset among different stations. In the OFDMA system orthogonality among sub-channels cannot be guaranteed if the clock offset is exceeded the threshold [2]. Therefore, we assume synchronization would be maintained efficiently to confine the maximum clock offset within the threshold, thereby ensuring the orthogonality among the OFDMA sub-channels.

\section{PROTOCOL IlLUSTRATION}

In this section, at first, we will discuss the method of subchannel distribution which is a complex procedure comparing to the pure random access mechanism. Then we will discuss the basic access mechanism and at last, we will discuss the advantages of our proposed MAC protocol.

\section{A. Sub-channel Distribution}

The main distinguishing feature of our hybrid protocol is its uniqueness in distributing the sub-channels to the terminals. As stated earlier, HTFA works in two steps: step 1 and step 2 where step 2 is conditional. In step 1 sub-channels are approximately 
evenly distributed to the terminals and in step 2 terminals within in a sub-channel will contend for medium randomly if the total number of terminals of the system is larger than the number of sub-channels. We said "approximately evenly distributed" because the number of terminals in the sub-channels is differed by at most one. We consider five distinguish scenarios for distributing the sub-channels:

Scenario 1: Number of sub-channels is equal to the number of terminals $(\mathrm{M}=\mathrm{N})$

Scenario 2: Number of sub-channels is greater than the number of terminals $(\mathrm{M}>\mathrm{N})$

Scenario 3: Some terminals leave the network early

Scenario 4: Number of terminals is greater than the number of sub-channels $(\mathrm{N}>\mathrm{M})$

Scenario 5: Some terminals join the network after some time

We describe each scenario as follow:

Scenario 1: Number of sub-channels is equal to the number of terminals $(M=N)$ : Suppose we have three terminals namely, Station A, Station B and Station C; and three subchannels namely, Sub-channel 1, Sub-channel 2 and Subchannel 3. Since in this case $M=N$ and the sub-channels are evenly distributed, each terminal will get exactly one subchannel (Fig. 3 (a)). It is not possible one terminal gets two subchannels and other two get one and zero terminal respectively. In this way, HTFA ensures fair access to the medium among the stations which is absent in the article [3] and [4].

Scenario 2: Number of sub-channels is greater than the number of terminals $(M>N)$ : Suppose we have two terminals Station A and Station B; and three sub-channels as mentioned above. Since in this case $M>N$ and sub-channels are approximately evenly distributed, one terminal gets two subchannels and other terminal gets one sub-channel i.e. Station A gets one sub-channel and Station B gets two sub-channels (Fig. 3 (b)).

Scenario 3: Some terminals leave the network early: Again, suppose at the beginning, Sub-channel 1, Sub-channel 2 and Sub-channel 3 are assigned to Station B, Station A and Station $\mathrm{C}$ respectively. After some time, Station B sent all of its data and releases its sub-channel i.e. Sub-channel 1. Now, one of the two remaining terminals (A or C) can acquire B's sub-channel and send data (Fig. 3 (c)).

Scenario 4: Number of terminals is greater than the number of sub-channels $(N>M)$ : Now suppose we have four terminals Station A, Station B, Station C and Station D; and three sub-channels as mentioned above. In this case, the first 3 terminals (A, B and C) are assigned to three sub-channels and the fourth terminal (Station D) is assigned to anyone subchannel. That means, two sub-channels get 1 terminal each and one sub-channel gets 2 terminals. Suppose, sub-channel 2 gets two terminals (Station A and Station D) which is shown in Fig. 4. Now that Station A and Station D are assigned to the same sub-channel (Sub-channel 2), they will randomly contend for channel access according to the legacy DCF (Distributed Coordination Function) rule.

Fig. 4 delineates random access procedure, where initially Station A and Station D generate random backoff number 5 and 7 respectively. As Station A generates a smaller number than Station D, Station A will access the sub-channel first when it's backoff counter reaches to zero. After one more slot time, D's backoff counter reaches to zero and thereby access the subchannel. In the second round, Station A and Station D generate new backoff value 5 and 2 respectively and the procedure will continue according to the DCF rule.

Scenario 5: Some terminals join the network after some time: How does a terminal join in the network after some time depends on the current status of the network. There are two statuses:

(i) $\quad \mathrm{M}>\mathrm{N}$

(ii) $\mathrm{N} \geq \mathrm{M}$

\begin{tabular}{|c|}
\hline $\begin{array}{c}\text { Sub-channel 1 } \\
\text { Station B }\end{array}$ \\
\hline $\begin{array}{c}\text { Sub-channel 2 } \\
\text { Station A }\end{array}$ \\
\hline Sub-channel 3 \\
Station C \\
\hline (a) \\
\hline Sub-channel 1 \\
Station B \\
\hline Sub-channel 2 \\
Station A \\
\hline Sub-channel 3 \\
Station B \\
\hline (b) \\
\hline Sub-channel 1 \\
\hline Station C \\
\hline Sub-channel 2 \\
Station A \\
\hline Sub-channel 3 \\
Station C \\
\hline (c) \\
\hline Station B \\
\hline
\end{tabular}

Fig. 3. Sub-channel distribution (a) Case 1 (b) Case 2 (c) Case 3 


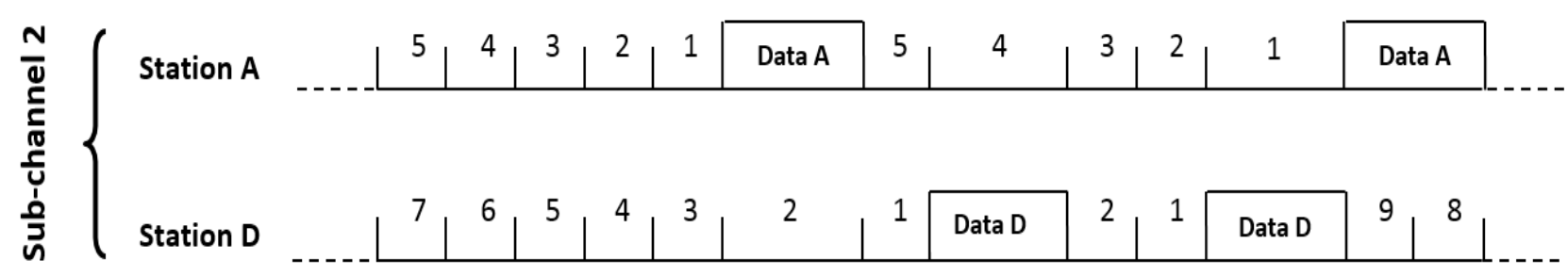

Fig. 4. The contention of two stations within a sub-channel

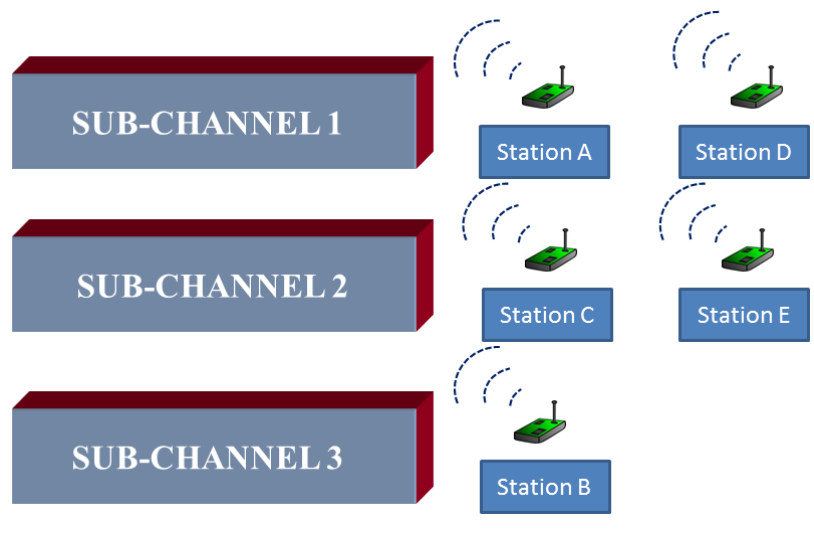

Fig. 5. Network configuration for 3 sub-channels and 5 stations

When $M>N$, then the new terminal will acquire a subchannel from the old terminal that has the highest number of sub-channels. The new terminal may need to wait for some time so that old terminal can send its ongoing packet. When $\mathrm{N} \geq \mathrm{M}$, then the new terminal will join a sub-channel that has the minimum number of terminals.

The sub-channel distribution procedure mentioned above is very intuitive. For further clarification of the procedure, we are going to demonstrate an example scenario. Suppose at the beginning there is an access point with no terminals at all and there are three sub-channels SUB-CHANNEL 1, SUBCHANNEL 2 AND SUB-CHANNEL 3. After some time, Station A joins the network and it will acquire all three subchannels. Then after some time Station B joins the network and it will seize one sub-channel (e.g. SUB-CHANNEL 3) from Station A. Now Station A has two sub-channels SUBCHANNEL 1 and SUB-CHANNEL 2; and Station B has one sub-channel (SUB-CHANNEL 3). Again, after some time Station $\mathrm{C}$ joins the network and it will seize one sub-channel (e.g. SUB-CHANNEL 2) from Station A. Now each of the three terminals has exactly one sub-channel. Suppose after some time Station D wants to join the network and the AP can put it to any sub-channel (e.g. SUB-CHANNEL 1) to contend for channel access by generating random back-off value. Similarly, Station $\mathrm{E}$ arrives and the AP assigns it to the sub-channel which has the minimum number of terminals. Now SUB-CHANNEL 2 and SUB-CHANNEL 3 has a minimum number of terminals (i.e., 1 terminal each) and suppose Station E got SUB-CHANNEL 2. Until now we get network configuration shown in Fig. 5, where SUB-CHANNEL 1 contains Station A and Station D; SUBCHANNEL 2 contains Station $\mathrm{C}$ and Station E; and SUBCHANNEL 3 contains Station B.
Now suppose Station E wants to leave the network. The subchannel distribution will not change after leaving Station E since the number of terminals is approximately evenly distributed. After a while, Station $\mathrm{C}$ also leave the network. Now the number of terminals is not approximately evenly distributed since SUB-CHANNEL 1 contains two terminals (Station A and Station D) and SUB-CHANNEL 2 contains no terminal. In this case, one terminal of SUB-CHANNEL 1 will migrate to SUB-CHANNEL 2. Thus, each sub-channel gets exactly one terminal and terminals are evenly distributed to the sub-channel.

\section{B. Access Mechanism}

A terminal with a new packet to transmit must be associated with the access point (AP). AP will assign sub-channels to the terminals according to the five scenarios mentioned in Section IV-A i.e. sub-channel distribution. Access method depends on the number of terminals available in a sub-channel. There would be two cases:

i. Multiple terminals in a sub-channel

ii. Single terminal in a sub-channel

i. Multiple terminals in a sub-channel: In this case, four-way handshaking (RTS/CTS, DATA/ACK) will be used as shown in Fig. 6 [22]. After DIFS (distributed inter-frame space) interval sending terminal enters into backoff interval. When backoff value reaches to zero, sending terminal transmits RTS (request-to-send) frame to the receiving terminal. After SIFS (short inter-frame space) interval receiver responds with the CTS (clear-to-send) frame. Upon receiving the CTS frame, the sender waits for the SIFS interval and then send the DATA frame. After receiving the DATA frame, the receiver again waits for SIFS interval and then responds with ACK (acknowledgement) frame.

In this case, all stations have to generate a random backoff time before sending data in order to minimize the probability of collision with the frames being sent by other stations in the same sub-channel. If two or more stations generate the same backoff number, a collision would occur and will lose data of the colliding stations as shown in Fig. 7.

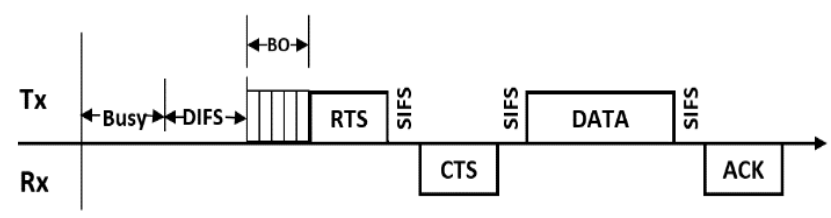

Fig. 6. Four-way handshaking for multiple stations in a sub-channel 


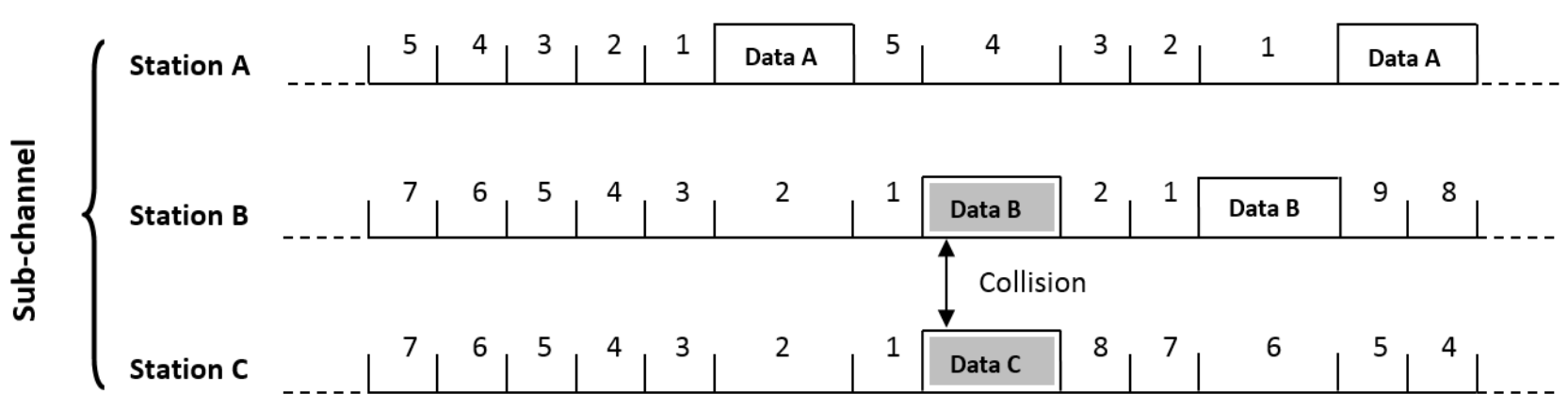

Fig. 7. A Collision between Station B and Station C in one particular sub-channel

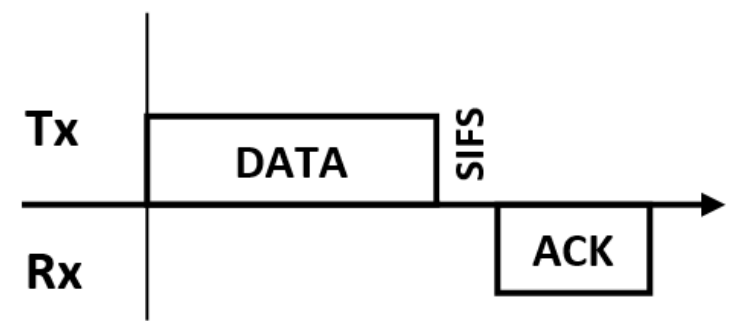

Fig. 8. Two-way handshaking for a single station in a sub-channel

Binary exponential backoff (BEB) algorithm determines the backoff time which is uniformly chosen in the range of $[0$, $\mathrm{W}-1$ ] for a contention window size $\mathrm{W}$. At the first transmission attempt $\mathrm{W}$ is set to the minimum value $\mathrm{W}_{\min }$ and is doubled at each backoff stage up to the maximum value $\mathrm{W}_{\max }=2^{\alpha} \mathrm{W}_{\min }$ after each unsuccessful transmission, where $\alpha$ denotes the number of backoff stages.

ii. Single terminal in a sub-channel: In this case, two-way handshaking (DATA/ACK) will be used as shown in Fig. 8. Since only one terminal monopolizes the sub-channel, there is no room for the hidden terminals to participate in the channel sharing. As a consequence, we need not the RTS/CTS frame pair. There is no DIFS and backoff interval as well which reduces overheads and increases throughput. In this case, the sending terminal first transmits the DATA to the receiving terminal. After waiting for a SIFS interval, the receiver sends the ACK frame to the sender.

\section{Advantages of HTFA}

Throughput Enhancement: The IEEE 802.11ax [also known as High Efficiency WLAN (HEW)] has a challenging goal of increasing the average throughput per user four times in highly dense environment. HTFA provides more throughput than several promising protocols which will be shown in Section VI. The terminals in HTFA will not contend for sub-channel access rather than sub-channels are dedicated to the terminals if $\mathrm{N} \leq$ $\mathrm{M}$. In this case, terminals monopolize the sub-channel access and there is no random backoff slot which significantly increases the throughput.

Collision Reduction: According to our model, a sub-channel could get at most one terminal when $\mathrm{N} \leq \mathrm{M}$. Thus, the probability of frame collision would be zero. Hence, the average throughput per terminal as well as total system throughput increases significantly. There is a probability of collision in some sub-channels if and only if $\mathrm{N}>\mathrm{M}$. However, in any case, the probability of collision in HTFA is smaller than any non-OFDMA channel (i.e. single channel).

Fair Access: Our proposed protocol employs a hybrid mechanism to distribute the sub-channels among the terminals. Thus, it not only provides a high throughput of data but also maintains improved fair access policy to the medium. The protocol works in two steps, where at the first step sub-channels are approximately evenly distributed to the terminals. Thus, the number of terminals in the sub-channels is differing by at most one. In the second step, terminals within a sub-channel will contend for medium randomly when $\mathrm{N}>\mathrm{M}$. Hence, HTFA performs better than SRMC-CSMA/CA and CM-CSMA/CA introduced in [4] and [3] respectively in terms of both throughput and fairness.

\section{ThOROUGHPUT ANALYSIS}

To measure the efficiency of a protocol, it is expected to validate the protocol employing an appropriate mathematical model that eventually increase the credibility and acceptability of the conducted research. Here, we will investigate the throughput of our proposed protocol using the analytical model presented in [12] by G. Bianchi. Bianchi formulates an ideal model for analyzing the saturated throughput of DCF which is followed by some other researchers. He designed the model using a discrete-time Markov chain, where the backoff mechanism is regulated by conventional single-channel CSMA/CA. Several other papers including [13] which are considered as extensions of [12] investigated the enhanced mathematical model for the actual backoff mechanisms by considering the existence of anomalous slots. Pioneer model in [12] and some of its extensions estimates saturation throughput of the single-channel terminal employing CSMA/CA mechanism. However, we will evaluate the saturation throughput of multi-channel terminals rather than the single channel which are also regulated by the CSMA/CA protocol.

Again, we assume that we have only one Basic Service Set (BSS) and the access point (AP) located at the centre of BSS. The BSS has $N$ terminals and $M$ sub-channels. We also assume that the number of terminals is very much larger than the number of subchannels i.e. $N \gg M$. The concept of saturated condition is the same as in [12] when WLAN carries the maximum load. We suppose that every station always has some packets available for sending. It implies the input queue of each terminal in WLAN is always non-empty in the saturated stage. 


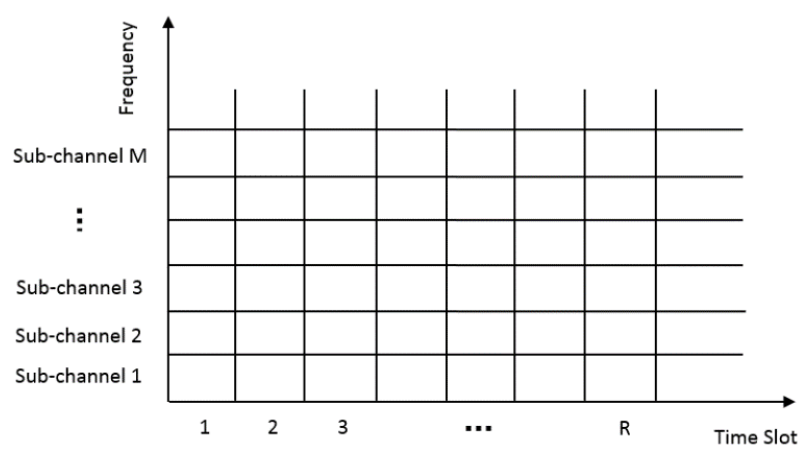

Fig. 9. Time-frequency block

It is not feasible to expand the Markov chain model of multichannel CSMA/CA from the traditional single channel-based model. Equations in [12] show that the author analyzed the characteristics of a single terminal in WLAN and received the transmission probability $(\tau)$ of a packet in a randomly chosen slot time. As a consequence, instead of directly evaluating the multichannel protocol model we will evaluate the probability of successful transmissions from the side of subchannels based on the random backoff mechanism.

In Fig 9 we defined a time-frequency block where each time slot in every sub-channel is considered as a resource utilized for acquiring the medium and transmission of data. We can find the number of time slots $(R)$ for the successful transmission of average packet payload $(E[p])$ as $R=T_{\text {total }} / T_{\text {slot }}$, where $T_{\text {total }}$ denotes the total average time and $T_{\text {slot }}$ denotes a single slot time. When a terminal gains a particular sub-channel at a random time slot then successful transmission interprets that only this particular terminal acquires this particular sub-channel for $R$ time slots. We can get the probability of successful access in one sub-channel at each time slice as $p_{k}=1 / R$ due to the equal probability of each single time slice under the presumption of the saturated stage.

We define the successful transmission probability $P_{j s u c}$ at the $j^{\text {th }}$ sub-channel when a terminal successfully utilizes one subchannel at one time slot as:

$$
P_{j s u c}=\sum_{k=0}^{R-1} p_{k}\left(\begin{array}{c}
N \\
1
\end{array}\right) \tau(1-\tau)^{N-1} .
$$

In the saturation stage, probability of collision $P_{j c o l}$ in one sub-channel must be:

$$
P_{j c o l}=1-P_{j s u c}
$$

Since each terminal can only gain one sub-channel at any time slot to send data at the saturated stage, the probability is dependent between $P_{1 s u c}$ and $P_{2 s u c}$. We can find the probability of $1^{s t}$ and $2^{\text {nd }}$ sub-channels having successful transmissions as below:

$$
\begin{aligned}
P(1 \text { suc, } 2 \text { suc }) & =\left.P_{1 \text { suc }} P_{2 s u c}\right|_{1 s u c} \\
& =P_{1 s u c} \sum_{k=0}^{R-1} p_{k}\left(\begin{array}{c}
N-1 \\
1
\end{array}\right) \tau(1-\tau)^{N-2} .
\end{aligned}
$$

We define $P_{s}(i)$ and $P_{c}(i)$ as there are $i$ sub-channels in our system having successful transmissions and collisions respectively.

Obviously $P_{s}(1)=P_{1 s u c}$. In general, the probability of $i$ subchannels having successful transmission computes as follow,

$$
\begin{aligned}
P_{s}(i)= & \left(\begin{array}{c}
M \\
i
\end{array}\right) P(1 s u c, 2 s u c, \ldots, i s u c) \\
& P((i+1) \operatorname{col},(i+2) \operatorname{col}, \ldots, M \mathrm{col}),
\end{aligned}
$$

We can get $P((i+1) \mathrm{col},(i+2) \mathrm{col}, \ldots, M \mathrm{col})$ following the procedure of equation (3).

As we assume $N \gg M, P_{1 s u c}$ and $P_{2 s u c}$ are independent and also equal to each other. Thus, $P(1 s u c, 2 s u c)$ could be rewritten as:

$$
P(1 s u c, 2 s u c)=P_{1 s u c} P_{2 s u c}=P_{1 s u c}^{2} .
$$

Simplifying equation (4) yields,

$$
\begin{aligned}
P_{s}(i)= & \left(\begin{array}{c}
M \\
i
\end{array}\right) P(1 \text { suc }, 2 s u c, \ldots, i s u c) \\
& P((i+1) c o l,(i+2) c o l, \ldots, M c o l), \\
= & \left(\begin{array}{c}
M \\
i
\end{array}\right) P_{1 s u c}^{i}\left(1-P_{1 s u c}\right)^{M-i},
\end{aligned}
$$

We can use $P_{S}(i)$ to find the average number of sub-channels $\left(E_{s}\right)$ having successful transmissions at any single time slot as below:

$$
E_{s}=\sum_{i=0}^{M-1} i P_{S}(i)
$$

Finally, we can obtain our desired saturation throughput $(S)$ as follow:

$$
S=\frac{E[p] \cdot E_{S}}{T_{\text {slot }}} .
$$

\section{Performance Evaluation ANd Simulation}

In this section, we first analyze the HTFA protocol with respect to different simulation parameters. After that, we compare and contrast the efficiency of HTFA (High Throughput and Fair Access) with some other promising protocols proposed by different researchers. All the simulation scenarios are implemented in 'Network Simulator-3'.

TABLE I

SIMULATION PARAMETERS FOR HTFA EVALUATION

\begin{tabular}{|l|l|}
\hline \multicolumn{1}{|c|}{ Parameters } & \multicolumn{1}{|c|}{ Value } \\
\hline Backoff slot duration & $50 \mu \mathrm{s}$ \\
\hline DIFS duration & $110 \mu \mathrm{s}$ \\
\hline Packet transmission time & $2.5 \mathrm{~ms}$ \\
\hline Minimum contention window size, $\mathrm{W}_{\min }$ & $32 \mathrm{slot}$ \\
\hline Number of backoff stages, $\alpha$ & 6 \\
\hline Number of stations, $\mathrm{N}$ & 10 \\
\hline
\end{tabular}




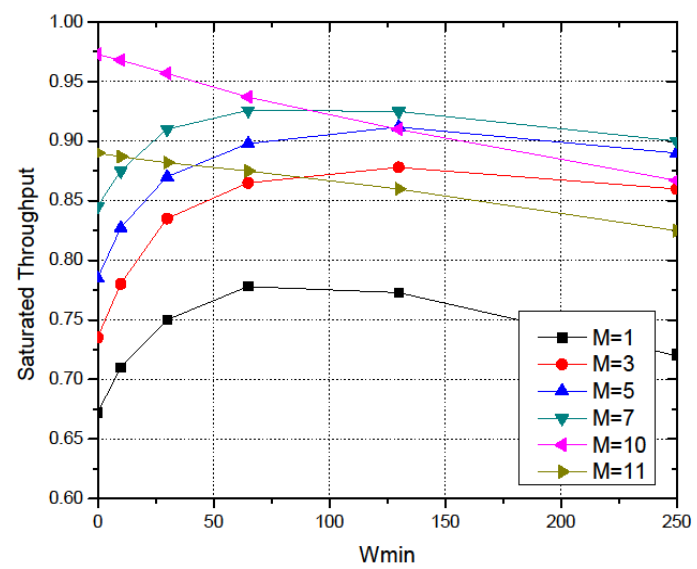

Fig. 10. Saturated throughput with respect to $\mathrm{W}_{\min }$ for the varying number of sub-channels

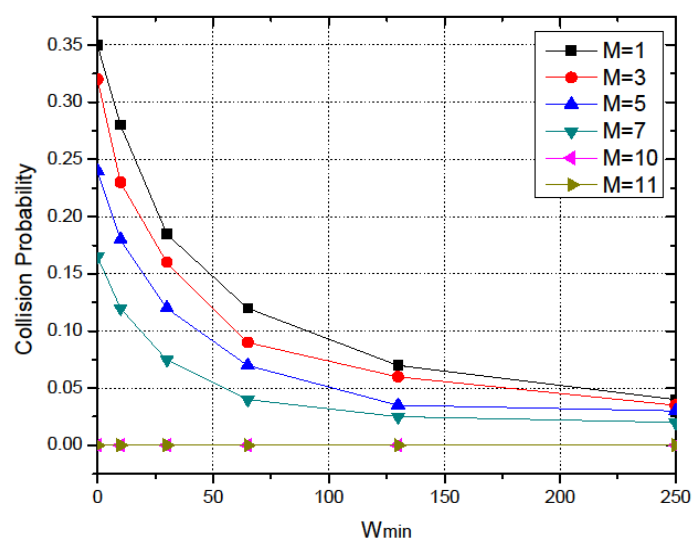

Fig. 11. Collision probability with respect to $\mathrm{W}_{\min }$ for the varying number of sub-channels

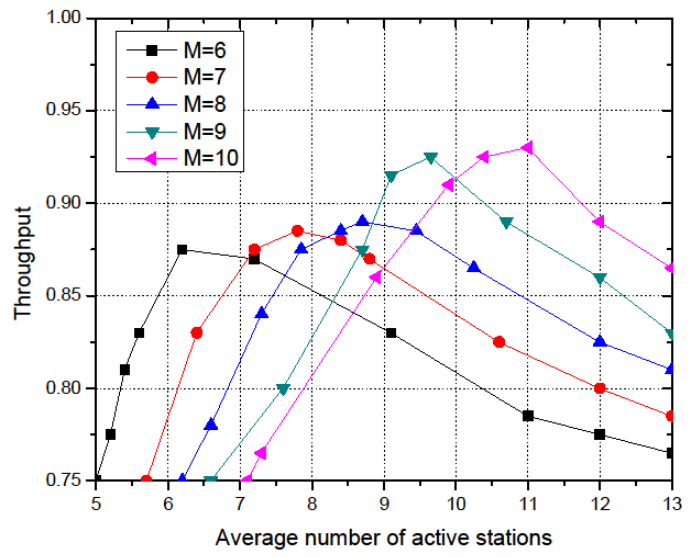

Fig. 12. Throughput in accordance with the average number of participating terminals under non-saturated traffic loads

\section{A. HTFA Evaluation}

We analyze the performance of our proposed OFDMA-based multi-channel hybrid protocol through comprehensive computer simulations. The simulation parameters are listed in Table I. In these experiments, we do not consider any transmission failure resulted from channel error.

Fig. 10 shows the saturated throughput of the HTFA protocol with respect to the minimum contention window size $\left(\mathrm{W}_{\min }\right)$ for the varying number of sub-channels. In this experiment we use 10 stations for different sub-channels i.e. $M=1,3,5,7,10$ and 11. The figure shows that maximum saturation throughput is enhancing gradually until the number of sub-channels increases to the number of stations. The saturation throughput decreases when the number of sub-channels exceeds the number of stations. This happens because the extra sub-channels are not utilized efficiently and there is also channelization overhead. We also observe irregular behaviour for $\mathrm{M}=10$ and 11 . Because in these cases, incrementing in the contention window size also increases the idle time while there is no significant reduction in collision probability. This incident suggests that when the number of sub-channels approaches the number of stations, we need not use a large time-domain backoff for collision resolution purpose as the collision probability would be very low in such stage.

Fig. 11 describes the collision probability of our multichannel protocol with respect to the minimum contention window size $\left(\mathrm{W}_{\mathrm{min}}\right)$ for varying numbers of sub-channels. The graphs show that collision probability decreases as $\mathrm{W}_{\text {min }}$ increases. It also reveals that collision probability is decreasing as the number of sub-channels is increasing for particular $\mathrm{W}_{\text {min }}$. It happens because increasing the number of sub-channels means less contention for channel access. It is observed that for $\mathrm{M}=10$ and 11 , the collision probability is zero because the number of stations (i.e. $\mathrm{N}=10$ ) is less than or equal to the number of sub-channels.

Now we evaluate the throughput performance of the proposed 'HTFA' hybrid protocol under non-saturated traffic loads. Fig. 12 shows the resulting throughput with respect to the average number of active stations. We find the similarly as reveals in saturated traffic load: the throughput enlarges until the number of active stations $\mathrm{N}$ exceeds the number of subchannels $\mathrm{M}$ but reduces gradually beyond that because collision occurs more frequently among the participating stations. As throughput loss resulted from extra sub-channels is larger than that caused by the collision, it is expected to keep the number of sub-channels slightly smaller than the average number of participating stations.

According to the above findings, we might adopt a system where the number of sub-channels would be fixed adaptively according to the number of participating stations. Specifically, we might incorporate an adaptive control mechanism in such a way that the access point (AP) first estimates the number of participating stations in the wireless LAN, then determines the number of optimum sub-channels from the estimation, and finally announces the result to the terminals through the control channel using beacon messages.

We further investigate the impact of increasing the backoff slot duration on the performance of the OFDMA-adopted multichannel hybrid system. We examine the saturation throughput of the OFDMA-employed wireless LAN with respect to the 


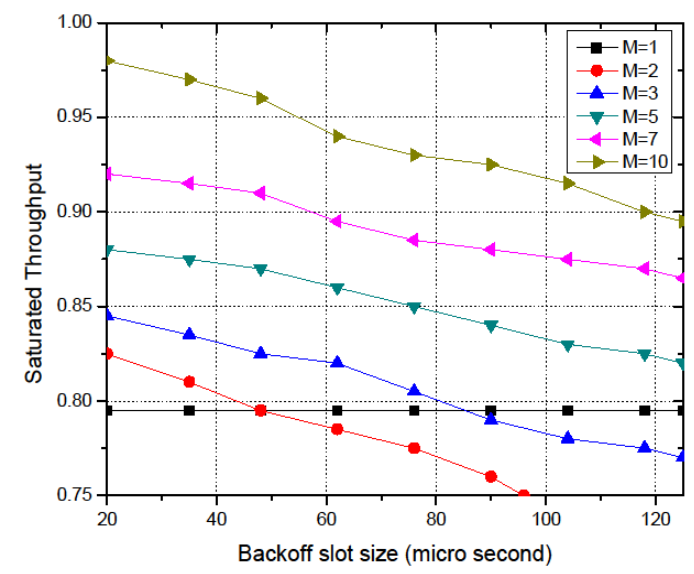

Fig. 13. Saturated throughput with respect to the backoff slot duration

TABLE II

SIMULATION PARAMETERS FOR PROTOCOL COMPARISON

\begin{tabular}{|l|l|}
\hline \multicolumn{1}{|c|}{ Parameters } & \multicolumn{1}{c|}{ Value } \\
\hline Slot time & $10 \mu \mathrm{s}$ \\
\hline Packet length & $1500 \mathrm{bytes}$ \\
\hline Total channel bandwidth & $54 \mathrm{Mbit} / \mathrm{sec}$ \\
\hline Number of stations & 3 \\
\hline Number of sub-channels & 3 \\
\hline $\mathrm{CW}_{\min }$ & 32 \\
\hline $\mathrm{CW}_{\max }$ & 1024 \\
\hline
\end{tabular}

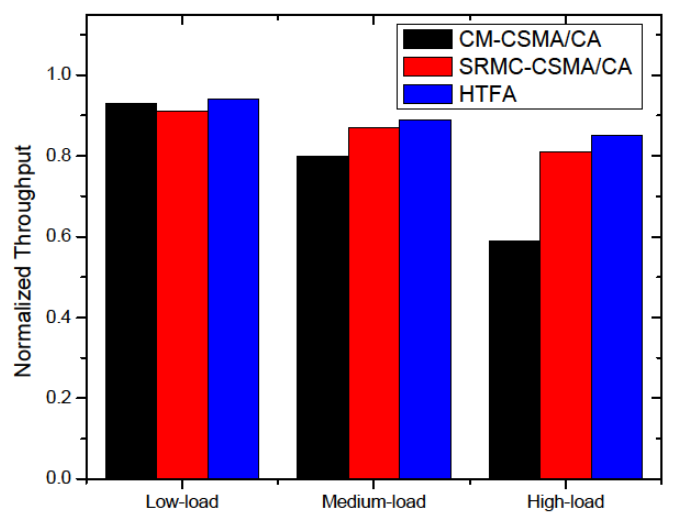

Fig. 14. Normalized throughput of stations having different traffic loads

TABLE III

TOTAL THROUGHPUT AND MAX-MIN FAIRNESS COMPARISON

\begin{tabular}{|c|c|c|c|}
\hline Metric & CM-CSMA/CA & SRMC-CSMA/CA & HTFA \\
\hline$T(\mathrm{Mbit} / \mathrm{sec})$ & 41.20 & 47.6 & 49.3 \\
\hline$F$ & 0.31 & 0.07 & 0.05 \\
\hline
\end{tabular}

backoff slot duration ranges from $20-120 \mu$ s. Fig. 13 shows the outcome where 10 stations actively participate. As we expected, the saturation throughput reduces as the backoff slot duration increases and vice-versa. Nevertheless, the OFDMA-employed multi-channel system still provides more throughput than the single-channel system up to a certain value of the backoff slot duration, for example, up to about 82 microseconds for $\mathrm{M}=3$ sub-channels. As the number of sub-channels increases, the outperforming range of the backoff slot duration increases and vice-versa.

\section{B. Protocol Comparison}

Here, we use the symbol $l_{i}$ to denote the traffic load and $t_{i}$ to denote achieved throughput of the $i^{\text {th }}$ terminal. Obviously, $t_{i} \leq l_{i}$. As traffic loads of different terminals may vary significantly, we wish to find normalized throughput $\left(t_{i} / l_{i}\right)$ for a fair comparison. We use two different metrics to examine the performance efficiency of our designed protocol. These two metrics are total throughput of the network denoted by $T$ and the max-min fairness denoted by $F$ which are measured according to the following equations:

$$
\begin{gathered}
T=\sum_{i=1}^{N} t_{i} \\
F=\max \frac{t_{i}}{l_{i}}-\min \frac{t_{i}}{l_{i}}
\end{gathered}
$$

Table II listed the simulation parameters for subsequent experiments. We assume terminals generate packets according to Poisson distribution and different terminals may have different traffic loads. We compare our HTFA protocol with the CM-CSMA/CA protocol proposed in [3] and SRMCCSMA/CA protocol proposed in [4] in the following scenario.

We conduct simulation for three terminals having loads 12 , 18 and 24 Megabits respectively and we referred those as low load, medium load and high load terminal. We assume total bandwidth of the whole channel is $54 \mathrm{Mbit} / \mathrm{sec}$ and the channel is evenly partitioned into three sub-channels. Thus, each of the sub-channels gains a bandwidth of $18 \mathrm{Mbit} / \mathrm{s}$. The normalized throughput of all terminals (low, medium and high load) is measured for the intended three protocols and is shown in Fig. 14. Analyzing the CM-CSMA/CA protocol, we see the normalized throughput reduces significantly with the increases in the traffic load. In CM-CSMA/CA protocol, one terminal can access only one sub-channel in most cases and hence the normalized throughput of the high load terminal does not exceed 0.60. On the other hand, according to HTFA and SRMC-CSMA/CA protocol, any terminal could acquire multiple sub-channels and transmits data concurrently. Therefore, the normalized throughput of the high load terminal is not bounded by the bandwidth of an individual sub-channel. We also observe that the normalized throughput of all the three types of loads is above 0.81 of HTFA and SRMC-CSMA/CA protocol. However, HTFA performs slightly better than SRMCCSMA/CA due to less contention and less collision ensured by HTFA.

The overall throughput and max-min fairness comparisons are summarized in Table III. The terminals in HTFA will not contend for sub-channel access rather than sub-channels are dedicated to the terminals if the number of terminals $(N)$ is smaller or equal to the number of sub-channels (M). If $\mathrm{N} \leq \mathrm{M}$, terminals monopolize the sub-channel access and there is no random backoff slot. Thus, HTFA provides higher throughput than CM-CSMA/CA and SRMC-CSMA/CA. Our HTFA protocol employs a hybrid mechanism to distribute the subchannels among the terminals. Thus, its max-min fairness is promising than CM-CSMA/CA and SRMC-CSMA/CA. 


\section{CONCLUSION}

The rapid growth of demand for high-speed WLAN has driven intense research to enhance the throughput by employing a variety of medium access control (MAC) mechanisms. The efficiency of MAC plays a major role to enhance the throughput of a Wireless LAN system. One of the promising access mechanisms for MAC is orthogonal frequency division multiple access (OFDMA). In this paper, we propose an OFDMA-based MAC protocol named 'HTFA' which employs a hybrid mechanism for channel access. HTFA will provide high throughput of data as well as maintains improved fair access policy to the medium among the terminals. The main distinguishing feature of our proposed protocol is its uniqueness in distributing the sub-channels to the terminals. We perform rigorous simulations with network simulator-3 that is presented in Section VI. Simulation results confirm validation of our protocol in terms of throughput, collision reduction and fairness. We get these advantages at the expense of increased complexity in the channel distribution procedure. Still, we convinced that there would be a tremendous tradeoff of the proposed protocol. Theoretical analysis of saturation throughput of the HTFA protocol is also evaluated in Section V employing an ideal comprehensive model.

Through simulation, we also able to approximate the number of sub-channels in accordance with the number of participating terminals in a wireless LAN where all sub-channels are equally dispersed. Throughout the article, we consider the sub-channels are of equal length to avoid complexity and to keep within the scope of the paper. However, it is also urged to establish a model and carry out simulations and mathematical analysis for varying sub-channel length which might be subject to future research.

\section{REFERENCES}

[1] Jing Wang, Guixia Kang and Ping Zhang, "A two-dimensional medium access control protocol based on OFDMA and CSMA/CA," Wireless Telecommunications Symposium (WTS), pp. 1-5, April 2011.

[2] Hojoong Kwon, Hanbyul Seo and Seonwook Kim, "Generalized CSMA/ CA for OFDMA systems: protocol design, throughput analysis, and implmentation issues," IEEE Transactions on Wireless Communications, Vol. 8, No. 8, pp. 4176-5187, August 2009.

[3] Xudong Wang and Hao Wang, "A novel random access mechanism for OFDMA wireless networks," Global Telecommunications Conference (GLOBECOM), Dec. 2010.

[4] Jia Xu, Pin Lv and Xudong Wang, "Single-radio multi-subchannel random access for OFDMA wireless networks," Electronics Letters, Vol. 49, No. 24, pp. 1574-1576, November 2013.

[5] Qiao Qu, Bo Li, Mao Yang and Zhongjiang Yan, "An OFDMA based concurrent multiuser MAC for upcoming IEEE 802.11ax," IEEE Wireless Communications and Networking Conference Workshops, March 2015 .

[6] Cao Xuelin, Song Zuxun and Yang Bo, "TR-MAC: A multi-step slot reservation-based hybrid MAC protocol for ad hoc networks," IEEE International Conference on Signal Processing, Communications and Computing (ICSPCC), pp. 1 - 5, Sept. 2015.

[7] Yunjie Yuan, Bo Li, Yi Chen and Hu Zhou, "CCRM: A MAC protocol with cooperative channel reservation for wireless ad hoc networks," 7th International Conference on Wireless Communications, Networking and Mobile Computing, pp. 1 - 4, Sept. 2011.

[8] Choi, Y. J., Park, S. and Bahk, S. "Multichannel random access in OFDMA wireless networks," IEEE Journal on Selected Areas in Comm. pp. 603-613 VOL. 24, NO. 3, March 2006.

[9] IEEE 802.11, Part 11: Wireless LAN medium access control (MAC) and physical layer (PHY) specifications, IEEE 802.11 Std., 2018.
[10] NS-3, "Network Simulator," https://www.nsnam.org/

[11] Md. Mustafizur Rahman, Choong Seon Hong and Sungwon Lee, "A high throughput on-demand routing protocol for multirate ad hoc wireless networks," IEICE Transactions on Communications, pp. 2939 VOL. E93-B, NO.1 January 2010.

[12] G. Bianchi, "Performance analysis of the IEEE 802.11 distributed coordination function," IEEE J. Select. Areas in Commun., vol. 18, no. 3, pp. 535-547, March 2000.

[13] I. Tinnirello, G Bianchi and Y. Xiao, "Refinements on IEEE 802.11 distributed coordination function modeling approaches," IEEE Transactions on Vehicular Technology, vol. 59, no. 3, pp. 1055-1067, March 2010.

[14] IEEE 802.11, Proposed 802.11ax Functional Requirements, IEEE 802.11-14/0567r7, July 2014.

[15] IEEE 802.11, HEW MAC Efficiency Analysis for HEW SG, IEEE 802.11-13/0505r0, May 2013.

[16] IEEE 802.11, Uplink Multi-user MAC Protocol for 11ax, IEEE 802.11-14/0598r0, May 2014

[17] IEEE 802.11, Discussion on OFDMA in IEEE 802.11ax, IEEE 802.1114/0839r1, Jul. 2014.

[18] "Introduction to 802.11ax High-Efficiency Wireless," White paper of 'National Instruments', June 2016. http://www.ni.com/white-paper/53150/en/

[19] Ian F. Akyildiz and Xudong Wang, "Wireless Mesh Networks" Wiley, pp. 52-53, 2009.

[20] G. Haile and J. Lim, "C-OFDMA: Improved throughput for next generation WLAN systems based on OFDMA and CSMA/CA," 4th International Conference on Intelligent Systems, Modelling and Simulation, pp. 497- 502, January 2013.

[21] H. Ferdous and M. Murshed, "Enhanced IEEE 802.11 by integrating multiuser dynamic OFDMA," Wireless Telecommunications Symposium (WTS), pp. 1-6, April 2010.

[22] Gazi Zahirul Islam and Mohammod Abul Kashem, "An OFDMAbased new MAC mechanism for IEEE 802.11ax," 5th International Conference on Networking, Systems and Security (NSysS), pp. 1-7, December 2018.

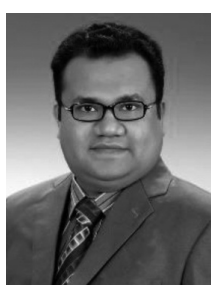

Gazi Zahirul Islam is currently pursuing his $\mathrm{PhD}$ at Bangladesh University of Professionals. He completed M.Sc. in wireless communications systems engineering from the University of Greenwich, UK and B.Sc. in computer science and engineering from Chittagong University of Engineering and Technology (CUET), Bangladesh. He has been teaching as an Assistant Professor at Daffodil International University, Bangladesh for about 5 years. Previously he taught at City University, Southern University Bangladesh and Primeasia Univesity. $\mathrm{He}$ is an author of several research articles on wireless communications and e-governance.

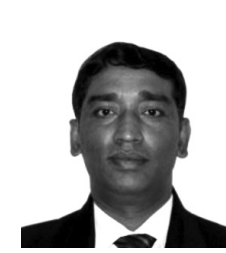

Mohammod Abul Kashem is a Professor of Computer Science and Engineering Department of Dhaka University of Engineering and Technology (DUET), Bangladesh. He also served as the director of "Institute of Information and Communication Technology" of DUET. He is a Post Doctorate of University Lumiera Lyon2, France and he completed $\mathrm{PhD}$ from National University "Lviv Politechnic" Ukraine. He also passed M.Sc. Engg. and B.Sc. Engg. from State University "Lvivska Polytechnica" Ukraine. His fields of specializations are mobile communication, information system management, cyber physical system and speech signal processing. He is an author of more than 50 articles on his research areas. 